\title{
Absenteísmo relacionado à doenças entre membros da eQuipe de enfermagem de um hospital escola
}

Disease-related absenteeism among nursing team members in a teaching hospital

Absentismo debido a las enfermedades entre los miembros del equipo de enfermería en un hospital de enseñanza

\author{
Fernanda Marques da Costa', Maria Aparecida Vieira", Roseni Rosângela de Sena'II \\ 'Estratégia Saúde da Família. Montes Claros, MG \\ "Universidade Estadual de Montes Claros. Montes Claros, MG \\ "IIUniversidade Federal de Minas Gerais. Belo Horizonte, MG
}

Submissão: $31 / 10 / 2007$

Aprovação: 10/1 1/2008

\section{RESUMO}

O artigo apresenta resultados da pesøuisa Que identificou causas do absenteísmo relacionado a doenças, justificado por atestado ou licença médica, entre os funcionários da enfermagem de um hospital público de Montes Claros - Minas Gerais. Trata-se de uma pesquisa descritivo-exploratória. Os resultados indicam Que os principais motivos de afastamento estão relacionados a problemas osteo-musculares e a enfermidades do aparelho respiratório; principalmente entre auxiliares de enfermagem do sexo feminino, casadas, com idade entre 25 e 45 anos, do turno diurno e com mais de um vínculo empregatício. Conclui-se pela necessidade de se desenvolver ações de prevenção e controle do absenteísmo como estratégia para a Qualidade de vida dos trabalhadores da enfermagem.

Descritores: Absenteísmo; Doenças profissionais; Enfermagem do trabalho.

\section{ABSTRACT}

The article presents results of the research that identified to causes of disease-related absenteeism, justified by medical license, among nursing employees of a public hospital in Montes Claros, MG, Brazil. This is a descriptive-exploratory study. Results indicate that the main reasons for absenteeism are related to musculoskeletal problems and to respiratory diseases; mainly between married female nurse aides, with age between 25 and 45 years, that works in day shift and with more than one employment. It was concluded that there is a necessity to develop prevention actions for controlling the absenteeism as a strategy for the Quality of life of nursing workers.

Descriptors: Absenteeism; Occupational diseases; Occupational health nursing.

\section{RESUMEN}

El artículo presenta los resultados de la investigación que hay identificado las causas del absentismo debido a enfermedades, las cuales tenían justificativa médica, entre los trabajadores de enfermería de un hospital publico de Montes Claros, MG, Brasil. Tratase de una investigación descriptiva-exploratoria. Los resultados indican Que las principales razones del absentismo están relacionados a problemas osteomusculares e a enfermedades respiratorias, principalmente entre los auxiliares de enfermería casadas del sexo femenino y Que trabajan en más de un empleo, con edad entre los 25 y 45 años, y Que trabajan en el periodo del día. Se concluye Que hay una necesidad de desarrollo de acciones de prevención para el control del absentismo como una estrategia para la calidad de vida de los trabajadores de enfermería.

Descriptores: Absentismo; Enfermedades profesionales; Enfermería del trabajo. 


\section{INTRODUÇÃO}

O trabalho sempre foi importante na vida das pessoas, seja como fator de crescimento e realização pessoal ou, em uma visão menos idealizada, como meio de sobrevivência. Por suas determinações históricas e econômicas, o trabalho pode ser compreendido como organizador da vida social, embora estabeleça caminhos para a dominação cultural, social e econômica e para a submissão do trabalhador ao capital. Na relação de subordinação, ocorre a desarticulação entre o pensar e o fazer. Para um conjunto de pessoas é reservado o direito de pensar e planejar; para outros cabe apenas a execução, sendo esses, via de regra, pertencentes à base da pirâmide social(1) .

A análise da relação capital/trabalho revela contradições, pois o mesmo trabalho que dignifica, confere status e reconhecimento ao ser humano, pode ser também fonte de sofrimento, de desequilíbrio físico e mental, de dor e frustração. Tudo isso se agrava com o estabelecimento do modelo capitalista e a exacerbação dos ditames da administração científica ${ }^{(2)}$. Nesse contexto, as condições de trabalho tornam-se insalubres, a ponto de agredir a dignidade dos trabalhadores, caracterizando uma prática laboral incompatível com a Qualidade de vida ${ }^{(3)}$.

Ao abordar o trabalho em instituições de saúde e relacioná-lo às condições ambientais, políticas e gerenciais, pode-se analisar a gênese de uma série de agravantes da integridade e da saúde do trabalhador. Os aspectos ambientais podem oferecer riscos de ordem física, Química, biológica, ergonômica, mecânica e psicológica. Assim, deve-se levar em conta a peculiaridade do ambiente hospitalar, onde os trabalhadores estão permanentemente em contato com o sofrimento e a morte ${ }^{(4)}$. Considera-se ainda Que a maioria das instituições de saúde possui estrutura políticoadministrativa pouco flexível, dificultando a autonomia dos trabalhadores. Mesmo com a utilização máxima de suas faculdades intelectuais e psicoafetivas de aprendizagem, os trabalhadores não conseguem adaptar-se à estrutura das instituições ${ }^{(5)}$.

Em virtude das condições desfavoráveis presentes no ambiente de trabalho, os trabalhadores acabam por buscar meios de compensar o sofrimento, tornando-se funcionários resistentes e adotando posturas defensivas. Tornam-se indiferentes ao processo laboral, optando pela fuga ao trabalho, Que pode ocorrer por meio de atestados, licenças médicas ou simplesmente por faltas injustificadas Que afetam os próprios trabalhadores, bem como as organizações, comprometendo os resultados finais dos serviços ${ }^{(1)}$.

Os parâmetros administrativos utilizados na gestão de recursos humanos e aplicados na maioria das instituições de saúde, são inespecíficos e inadequados, contribuindo para a fuga do trabalho. Esse processo pode ser compreendido como o hábito de faltar ao trabalho voluntária ou involuntariamente, constituindo-se na somatória dos períodos em Que os empregados encontram-se ausentes ao trabalho, seja por falta ou atraso ${ }^{(6)}$.

Absenteísmo é um termo abrangente, Que pode assumir uma série de significações, conforme a abordagem que se faz. O fenômeno será considerado neste artigo, como a ausência ao trabalho relacionada a doença justificada por atestado caracterizado por afastamento de um a três dias, sem comprometimento dos proventos salariais e livre de prejuízos na contagem do tempo de serviço do trabalhador ${ }^{(7)}$. Por licença médica, denomina-se o afastamento superior a três dias, Que pode ser de curto prazo, com tempo igual ou inferior a 15 dias e de longa duração, aquelas em Que o prazo de afastamento supera 15 dias ${ }^{(8)}$. Tanto os atestados Quanto as licenças seguem os critérios de avaliação e consentimento médicos, face à constatação de incapacidade para o trabalho ${ }^{(8)}$. Neste estudo, não foram consideradas as faltas devidas a acidentes de trabalho, por se tratar de uma temática Que merece estudo específico. Não foi, considerado o absenteísmo voluntário, Que ocorre em razão de motivos particulares do funcionário, nem o absenteísmo legal amparado por lei como: gestação, nojo, gala, doação de sangue e serviço militar. Também não foi considerado o absenteísmo relacionado ao atraso ou abandono do serviço antes do cumprimento da carga horária, nem o absenteísmo devido a férias ou folgas, por serem essas consideradas pela Organização Internacional do Trabalho ${ }^{(8)}$ como ausências previstas e passíveis de planejamento.

A enfermagem é responsável pelo maior contingente da força de trabalho dos estabelecimentos hospitalares, com responsabilidade pela assistência e gestão nas 24 horas. É o conjunto de trabalhadores Que mais sofre com a inadequada condição de trabalho e com a insalubridade do ambiente. Pelo prisma da gerência de recursos humanos, o trabalhador e o próprio trabalho são atingidos pelas medidas de redução de $\operatorname{custos}^{(9)}$.

O absenteísmo na equipe de enfermagem é um problema relevante, considerando-se Que a maioria das instituições, inclusive os hospitais, não prevê cobertura para os dias de falta. A sobrecarga de trabalho para a equipe de enfermagem pode, inclusive, comprometer a Qualidade da assistência prestada à clientela ${ }^{(10)}$.

A Recomendação ${ }^{\circ} 171$ e a convenção $n^{\circ} 161$ da Organização Internacional do Trabalho (OIT) evidenciam a importância de registrar as causas do absenteísmo, a fim de se obter dados para a realização de análises Que contribuirão para conhecer a dimensão, as determinações e causas do absenteísmo e propor soluções ${ }^{(11)}$.

Nessa perspectiva, esta investigação possibilita reflexão sobre a magnitude do problema e poderá subsidiar ações de controle do absenteísmo na equipe de enfermagem de um Hospital Público de Montes Claros - Minas Gerais, contribuindo para a criação de novas possibilidades de tratar o fenômeno. Espera-se Que os resultados estimulem reflexões objetivando esforços para a obtenção de uma organização de trabalho Que elimine ou minimize os efeitos nocivos à saúde dos trabalhadores: enfermeiro, técnico de enfermagem e auxiliar de enfermagem.

São objetivos específicos deste estudo: traçar o perfil dos funcionários atingidos pelo absenteísmo relacionado a doença, justificado por atestado ou licença médica; especificar as principais doenças relacionadas nos atestados ou licenças médicas e identificar as variáveis relacionadas como causas das faltas ao trabalho.

Após delinear o problema e traçar os objetivos, com base na literatura pesQuisada, propõem-se as seguintes hipóteses: primeira hipótese: sugere-se Que, devido às más condições de trabalho, à jornada diária, ao trabalho em turnos, à realização de horas extras e ao desenvolvimento das tarefas em ritmo acelerado ${ }^{(4)}$, os trabalhadores da enfermagem desenvolvem doenças provenientes do cansaço físico e mental, sendo as do sistema ósteo-muscular a primeira causa de afastamento.

Segunda hipótese: os trabalhadores Que mantêm dupla ou tríplice 
jornada de trabalho, sujeitos a maiores exigências físicas, mentais, intelectuais e emocionais, têm maior número de faltas, se comparados aos Que conservam apenas um vínculo empregatício.

Terceira hipótese: o funcionário Que trabalha no turno noturno está mais sujeito a se ausentar do trabalho, se comparado com os Que trabalham durante o dia, devido à sua dificuldade de adaptação ao horário, o Que provoca alteração do ritmo biológico e causa desorganização do padrão de sono e repouso, bem como acarreta dificuldades na vida familiar e social ${ }^{(11)}$.

Quarta hipótese: em razão de as mulheres serem submetidas a uma jornada prolongada de trabalho, sendo responsáveis pelos afazeres domésticos, pelo cuidado dos filhos e também por enfrentarem disparidades no mercado de trabalho - diferenças salariais - são mais suscetíveis ao desgaste físico e mental e conseqüente adoecimento, incorrendo, assim, com maior freeüência, em faltas ao trabalho, se comparadas aos funcionários do sexo masculino ${ }^{(12)}$.

Quinta hipótese: os trabalhadores de nível fundamental e médio ausentam-se mais do trabalho do que os de nível superior.

\section{METODOLOGIA}

Trata-se de uma pesquisa de caráter descritivo, pois descreve as características relacionadas ao absenteísmo buscando estabelecer suas relações com variáveis pré-definidas. É, ainda, uma pesQuisa documental, pois procurou revisar dados areuivados - atestados e licenças médicas - localizados no Setor de Pessoal do Hospital.

Nos atestados e licenças médicas analisados estavam especificados os diagnósticos das doenças Que motivaram o afastamento, expressos na Classificação Internacional de Doenças (CID), a Qual garante ao profissional o direito ao sigilo e permite Que os documentos sejam analisados com a devida confidencialidade.

A população-alvo da pesquisa constituiu-se de todos os membros da equipe de enfermagem Que recorreram a atestados e licenças médicas de janeiro de 2000 a agosto de 2004. Foram encontrados 764 documentos, entre atestados e licenças, Que correspondem a 189 funcionários. Dos 189 funcionários, foram excluídos da pesquisa 46, sendo nove Que estavam em férias no período da coleta dos dados; sete Que estavam em licença para tratamento de saúde; Quatro Que estavam em licença maternidade; dois Que se recusaram a responder o Questionário e 24 Que já não trabalham mais no Hospital.

Assim, foram considerados, para este estudo, os funcionários Que recorreram a atestados e/ou licenças médicas, Que não se enQuadraram nos critérios de exclusão e Que estavam trabalhando no período de coleta dos dados, perfazendo um total de 143 profissionais, dos Quais foram encontrados 565 registros de afastamento.

A coleta de dados foi manual, realizada nos atestados e licenças médicas disponíveis no Setor de Pessoal, onde foram catalogados os dados referentes à especificação da Classificação Internacional de Doenças, presente nas cópias dos atestados e licenças médicas e que foram agrupados conforme a categoria profissional - auxiliar de enfermagem, técnico de enfermagem e enfermeiro. Cada licença ou atestado analisado recebeu uma codificação numérica, para garantir o anonimato dos envolvidos no estudo (143). Utilizou-se, também, o Questionário para traçar o perfil dos 143 trabalhadores, contendo as seguintes variáveis: categoria profissional - enfermeiro, técnico de enfermagem ou auxiliar de enfermagem -, sexo, faixa etária, estado civil, presença de filhos, turno, setor de trabalho e a presença de vínculo empregatício com uma ou mais instituições, além do hospital em estudo. O Questionário foi submetido a um pré-teste para se verificar sua adequação.

A análise dos dados foi realizada por meio do Microsoft Excel for Windows 2000 e SPSS 11 . for Windows 2001. Também foram realizados cálculos estabelecidos por estudiosos do processo de absenteísmo como Chiavenato $^{(6)}$, Silva e Marziale ${ }^{(12)}$ e Mendes $^{(1)}$.

Conforme prevê a Resolução n 196/96, o projeto de pesquisa foi submetido à apreciação do Comitê de Ética e Pesquisa (CEP) competente, sendo aprovado pelo Parecer Consubstanciado $n^{\circ} 122 / 4$ de 27 de outubro de 2004. Ressalta-se Que a Coordenadora de Enfermagem do Hospital assinou um Termo de Consentimento e Compromisso e os informantes assinaram um Termo de Consentimento Livre e Esclarecido, confirmando sua participação voluntária, sem benefícios e prejuízos.

A análise dos dados foi realizada por meio da estatística descritiva, utilizando-se gráficos e tabelas para apresentar os dados e a interpretação estatística com o Software SPSS II for Windows.

\section{RESULTADOS}

De acordo com as características profissionais da equipe de enfermagem do hospital cenário do estudo, representados na Tabela 1 , verificou-se o perfil dos funcionários Que recorreram a atestados e/ou licenças médicas no período de 2000 a 2004, Que se encontram distribuídos da seguinte forma: predominância da categoria auxiliar de enfermagem $(61,5 \%)$, sendo em sua maioria mulheres (81 , 1\%), na faixa etária de 36 a 45 anos $(40,6 \%)$, casados $(67,1 \%)$, possuindo filhos $(75,9 \%)$.

Verifica-se na Tabela 1 como os funcionários Que recorreram a licença e/ou atestado encontram-se distribuídos, segundo diferentes variáveis.

Dos 565 afastamentos distribuídos entre licenças e atestados no período estudado não se observam grandes variações, sendo a incidência de absenteísmo Quase Que constante.

Os dados da Tabela 2 revelam Que, dos 565 afastamentos 56,8.\% (32I) ocorreram entre auxiliares de enfermagem, 29,7\% (168) entre técnicos de enfermagem e 13,5\% (76) entre enfermeiros. Em estudos semelhantes, outros autores ${ }^{(13)}$ encontraram $67 \%$ em afastamentos dos auxiliares de enfermagem.

Neste estudo, verificou-se Que dos 565 atestados e licenças, 489 correspondem a auxiliares e técnicos de enfermagem e 76 aos enfermeiros, ou seja, a incidência de faltas dos auxiliares e técnicos de enfermagem é seis vezes maior Que o dos profissionais de nível superior. Estudos realizados por Alves ${ }^{(3)}$ apontam que o contingente de faltas entre os auxiliares e técnicos de enfermagem pode ser até três vezes maior Que o dos enfermeiros.

Os enfermeiros foram responsáveis por 13,5\% (76) dos afastamentos. Reis e La Rocca ${ }^{(13)}$ afirmam Que a menor freeüência de afastamentos de enfermeiros no trabalho pode ser atribuída ao fato de Que, sendo o profissional Que responde pela equipe de enfermagem, a responsabilidade do cargo pode determinar uma presença mais constante. Os dados mostram Que os afastamentos 
Tabela 1. Características dos profissionais da equipe de enfermagem pesquisados segundo variáveis. Montes Claros, 2004.

\begin{tabular}{|c|c|c|}
\hline VARIÁVEIS & $\mathrm{n}$ & $\%$ \\
\hline \multicolumn{3}{|l|}{ Categoria profissional } \\
\hline Enfermeiro & 13 & 9,1 \\
\hline Técnico de Enfermagem & 42 & 29,4 \\
\hline Auxiliar de Enfermagem & 88 & 61,5 \\
\hline \multicolumn{3}{|l|}{ Sexo } \\
\hline Masculino & 27 & 18,9 \\
\hline Feminino & 116 & 81,1 \\
\hline \multicolumn{3}{|l|}{ Faixa etária } \\
\hline $18-25$ & 3 & 2,1 \\
\hline $26-35$ & 53 & 37,1 \\
\hline $36-45$ & 58 & 40,6 \\
\hline Mais de 45 & 29 & 17,5 \\
\hline \multicolumn{3}{|l|}{ Estado Civil } \\
\hline Solteiro & 36 & 25,2 \\
\hline Casado & 96 & 67,1 \\
\hline Divorciado & 9 & 6,3 \\
\hline Viúvo & 2 & 1,4 \\
\hline \multicolumn{3}{|l|}{ Presença de Filhos } \\
\hline Não & 33 & 23,1 \\
\hline I filho & 34 & 23,8 \\
\hline 2 filhos & 44 & 30,1 \\
\hline 3 ou mais & 32 & 22,4 \\
\hline \multicolumn{3}{|l|}{ Turno de trabalho } \\
\hline Diurno & 94 & 65,7 \\
\hline Noturno & 49 & 34,3 \\
\hline \multicolumn{3}{|l|}{ Existência de duplo vinculo empregatício } \\
\hline Apenas o Hospital cenário & 79 & 52,2 \\
\hline em mais de I instituição & 62 & 42,7 \\
\hline em mais de 2 instituições & 2 & 1,4 \\
\hline
\end{tabular}

Tabela 2. Afastamentos de acordo com categoria profissional. Montes Claros, 2004.

\begin{tabular}{lcc}
\hline \multirow{2}{*}{$\begin{array}{c}\text { CATEGORIA } \\
\text { PROFISSIONAL }\end{array}$} & \multicolumn{2}{c}{ ATESTADOS E/OU LICENÇAS MÉDICAS } \\
\cline { 2 - 3 } & $\mathrm{n}$ & $\%$ \\
\hline Enfermeiro & 76 & 13,5 \\
Técnico de Enfermagem & 168 & 29,7 \\
Auxiliar de Enfermagem & 321 & 56,8 \\
\hline Total & 565 & 100,0 \\
\hline
\end{tabular}

ocorridos são mais facilmente resolvidos por arranjos internos entre trabalhadores hierareuicamente superiores, o Que pode ocasionar um menor índice de afastamentos por adoecimento nessa categoria.

A ocorrência de uma maior Quantidade de atestados entre os auxiliares de enfermagem está de acordo com os achados da literatura. Os estudos de Alves e Godoy ${ }^{(3)}$ demonstram Que quanto mais baixo o nível hierárQuico ocupado pelos trabalhadores da equipe de enfermagem, maior a probabilidade de afastamentos por motivo de adoecimento. Tal fato talvez se explieue pela natureza do trabalho desenvolvido pelo auxiliar de enfermagem: tarefas Que exigem maior esforço físico, atividades repetitivas e monótonas, contato muito próximo com o sofrimento ${ }^{(9)}$. Estudos de Nascimento $^{(9)}$ e Silva e Marziale ${ }^{(12)}$ abordando o absenteísmo relacionado a doença em uma equipe de enfermagem, mostraram Que 65\% das ausências ocorriam entre os auxiliares de enfermagem, assemelhando-se aos resultados deste estudo.

Considerando Que, para esses 143 profissionais a falta ao trabalho é uma constante, procurou-se associar a ocorrência do absenteísmo por doença com as variáveis dependentes consideradas nesta pesquisa - categoria profissional, sexo, faixa etária, estado civil, Quantidade de filhos, turno, setor de trabalho e existência de duplo ou triplo vínculo empregatício. 
Tabela 3. Ocorrência de absenteísmo dos profissionais da equipe de enfermagem segundo o setor de trabalho. Montes Claros, 2004.

\begin{tabular}{|c|c|c|}
\hline SETOR & $n$ & $\%$ \\
\hline Unidade de Tratamento Intensivo (UTI) Neonatal & 23 & 16,1 \\
\hline Clinica Médica B (CMB) & 18 & 12,6 \\
\hline Clínica Médica A (CMA) & 17 & 11,9 \\
\hline Pediatria & 15 & 10,5 \\
\hline Bloco Obstétrico & 13 & 9,1 \\
\hline Maternidade & 12 & 8,4 \\
\hline Central de Material Esterilizado (CME) & 10 & 7,0 \\
\hline Bloco Cirúrgico & 8 & 5,6 \\
\hline Berçário & 8 & 5,6 \\
\hline Clinica Cirúrgica & 7 & 4,9 \\
\hline Pronto Atendimento & 7 & 4,9 \\
\hline Supervisão & 4 & 2,8 \\
\hline Grupo de Apoio Nutricional & 1 & 0,7 \\
\hline TOTAL & 143 & 100,0 \\
\hline
\end{tabular}

Tabela 4. Ocorrência de Doenças Agrupadas pela CID* dos funcionários da equipe de enfermagem do Hospital cenário, conforme o sexo. Montes Claros, 2004.

\begin{tabular}{lcccccc}
\hline GRUPO DO CID & MASCULINO & \% & FEMININO & \% & TOTAL & \% \\
\hline Doenças do Aparelho Respiratório & 6 & 10,8 & 75 & 14,9 & 81 & 14,3 \\
Doenças do Sistema Osteomuscular e Conjuntivo & 16 & 28,6 & 119 & 23,5 & 135 & 24,0 \\
Doenças do Aparelho Digestivo & 4 & 7,4 & 29 & 5,7 & 33 & 5,8 \\
Transtornos Mentais e Comportamentais & 5 & 8,9 & 22 & 4,4 & 27 & 4,7 \\
Doenças do Aparelho Circulatório & 3 & 5,3 & 45 & 8,8 & 48 & 8,5 \\
Doenças do Aparelho Geniturinário & 2 & 3,5 & 35 & 6,8 & 37 & 6,5 \\
Gravidez, Parto e Puerpério & 0 & 0,0 & 44 & 8,6 & 44 & 7,8 \\
Doença da Pele e Anexos e Subcutâneo & 2 & 3,5 & 30 & 5,8 & 32 & 5,7 \\
Doenças dos Olhos e Anexos & 2 & 3,5 & 14 & 2,7 & 16 & 2,7 \\
Doenças Infecciosas e Parasitárias & 5 & 8,9 & 22 & 4,4 & 27 & 4,7 \\
Doenças do Ouvido & 0 & 0,0 & 5 & 0,9 & 5 & 0,9 \\
Doenças Nutricionais e Metabólicas & 0 & 0,0 & 5 & 0,9 & 5 & 0,9 \\
Doenças do Sistema Nervoso & 3 & 5,3 & 11 & 2,2 & 14 & 2,8 \\
Causas Externas de Morbidade & 2 & 3,5 & 9 & 1,8 & 11 & 1,9 \\
Fatores Que Induziram a Procura de Serviço de & 6 & 10,8 & 42 & 8,2 & 48 & 8,5 \\
Saúde não Especializado & & 0,0 & 2 & 0,4 & 2 & 0,2 \\
Neoplasias & & 100,0 & 509 & 100,0 & 565 & 100,0 \\
\hline TOTAL DE OCORRÊNCIA POR SEXO & 56 & & &
\end{tabular}

Na Tabela I os dados demonstraram a predominância do sexo feminino nos casos de absenteísmo, sendo Que as mulheres representam $81,1 \%$ (1 16) e os homens 18,9\% (27) do total da população do estudo Reis e La Rocca ${ }^{(13)}$, ao estudar o absenteísmo entre os profissionais da equipe de enfermagem em um hospital de médio porte, encontraram Que $81,4 \%$ dos afastamentos do trabalho ocorriam com mulheres. Após analisar a concessão de licenças médicas aos funcionários de um hospital-escola, Alves e Godoy ${ }^{(3)}$ também verificaram que 80, $1 \%$ das licenças concedidas foram para mulheres. Resultados semelhantes foram relatados por Silva e Marziale(12) $^{(12 u e}$ identificaram uma freqüência de $89,9 \%$ de afastamentos entre as mulheres ${ }^{(12)}$.

Alguns autores ${ }^{(11-13)}$ relacionam, como causa do absenteísmo feminino, o fato de a maioria das mulheres inseridas no mercado de trabalho serem ainda responsáveis pelos afazeres domésticos e cuidados aos filhos, geralmente, chegando ao serviço cansadas pela fadiga residual e pelo trabalho Que já realizaram em casa.
Essa dualidade de papéis da mulher repercute em seu cotidiano e durante o seu turno de trabalho, não conseguindo afastar-se dos problemas do lar.

Ressalta-se Que, na enfermagem, a predominância feminina é decorrente de fatores históricos. A divisão social do trabalho impôs à mulher as atividades de cuidado a doentes, crianças e idosos. Como o hospital era considerado espaço privilegiado para a profissionalização do trabalho doméstico, as mulheres foram assumindo esse campo, principalmente como profissionais da enfermagem. Assim, o trabalho doméstico foi incorporado ao trabalho em saúde. Mantiveram-se, para as mulheres, as características de subordinação e de mão-de-obra barata com caráter servil. Os reflexos estão nos salários mais baixos e nas duplas jornadas ${ }^{(3)}$.

No caso das mulheres, o trabalho em turnos traz conseqüências importantes para sua Qualidade de vida. Forçadas a cumprir dupla jornada de trabalho, têm o descanso após o turno dificultado, por 
ter Que assumir os encargos domésticos e o cuidado aos filhos. Por isso, adoecem mais freqüentemente e faltam mais ao trabalho ${ }^{(1,3)}$.

Na Tabela 1 também é possível observar Que a faixa etária da população em estudo: $43,4 \%$ (62) encontram-se entre 38 e 45 anos; 37,1\% (53) entre 26 e 27; 17,5\% (25) mais de 45 anos e $2,1 \%$ (3), estão com idade entre 18 e 25 anos.

Os resultados encontrados neste estudo assemelham-se aos encontrados em pesquisas realizadas por Silva et $\mathrm{al}^{(12,13)}$ Que, buscando relacionar as características da categoria e ausência ao trabalho, encontraram predomínio de afastamentos na faixa etária de 21 a 45 anos. As autoras atribuem esse resultado ao fato de a maioria dos profissionais de enfermagem serem mulheres, Que estão em idade reprodutiva e apresentam maior índice de absenteísmo por complicações na gravidez e no puerpério, além de estarem sobrecarregadas pelo cuidado aos filhos e afazeres domésticos. Os achados são condizentes com os desta pesquisa, Que evidenciou uma freqüência de $81,1 \%$ dos afastamentos entre as mulheres, com $77 \%$ dos afastamentos ocorrendo entre profissionais na faixa etária de 26 a 45 anos. Nascimento ${ }^{(9)}$ registrou uma maior freqüência de afastamentos na faixa etária de 40 a 50 anos $(86,4 \%)$; associando o achado ao fato de Que, conforme o avanço da idade, há também uma maior probabilidade dos trabalhadores apresentarem morbidades.

Quanto ao estado civil, verifica-se Que dos 143 funcionários faltosos, 67,1\% (96) são casados e 25,2\% (36) solteiros. Outros, $6,3 \%$ (9) e 1,4\% (2) são, respectivamente, divorciados e viúvos. Ao abordar o absenteísmo relacionado ao adoecimento e estado civil, Alves; Alves e Godoy ${ }^{(1,3)}$ afirmam Que os trabalhadores casados apresentam maior índice de afastamentos por terem mais responsabilidades domésticas. Corroborando os achados deste estudo, outra pesquisa mostrou Que 52,3\% dos profissionais envolvidos em faltas são casados ${ }^{(12)}$.

Ao abordar a variável Quantidade de filhos a TAB. I demonstra Que, dos 143 Que se afastaram do trabalho, 30,8\% (44) têm 2 (dois) filhos; 23,8\% (34) têm I (um) filho; 22,4\% (32) com 3 (três) filhos ou mais, sendo Que os 23,1\% (33) restantes não possuem filhos

Os dados evidenciam Que $77 \%$ dos funcionários pesquisados possuem filhos, enquanto Que $23,1 \%$ não os possuem e, dessa forma, foi possível relacionar a presença de filhos ao absenteísmo por doença. Os estudos de Nascimento; Mendes ${ }^{(9,11)}$ encontraram relação entre o fato de ter filhos e o maior índice de faltas ao trabalho por motivo de doença. Essa relação fundamenta-se em razão de Que muitas vezes o cônjuge do/a funcionário/a também trabalha fora, e não há com Quem deixar as crianças, ou mesmo porQue a preocupação com os cuidados dos filhos e a saúde dos mesmos pode representar uma sobrecarga mental para o funcionário, levando-o a ausentar-se por motivos de saúde. Nesse sentido, Silva e Marziale ${ }^{(12)}$ encontraram maior freeüência, 59\%, de faltas ao trabalho por motivo de doença entre as funcionárias Que tinham filhos.

Os dados da Tabela I evidenciam ainda Que, dos profissionais peseuisados, $65,7 \%$ (94), trabalhavam no turno diurno e $34,3 \%$ (49) no noturno.

Ao relacionar o absenteísmo por motivo de adoecimento e o turno de trabalho, esperava-se Que os trabalhadores do período noturno se afastem mais, porque o trabalho noturno provoca alterações fisiológicas decorrentes da ausência de sincronismo entre seu rítmo circadiano e o prolongamento do período de vigília. Mendes $^{(1)}$ assegura Que, devido ao limite entre vigília e repouso enfrentado pelos trabalhadores durante uma noite de trabalho, pode haver comprometimento da capacidade de concentração e, além disso, esses funcionários sofrem a privação do convívio com a família em razão da incompatibilidade de horário. Assim, o conjunto desses fatores pode desencadear problemas de ordem emocional e psíquica. Neste estudo, porém, não foi observada maior freQüência de faltas entre os funcionários do turno noturno ${ }^{(1)}$.

Na Tabela 3 verifica-se Que, dentre os trabalhadores Que faltaram ao trabalho por motivo de doença, a maioria está lotada na Unidade de Tratamento Intensivo, 16,1\%(23); seguida da Clinica Médica B, 12,6\%(18) e de $11,9 \%$ (17) na Clinica Médica A.

A literatura revisada evidencia maior freqüência de faltas relacionadas a doença entre os trabalhadores da equipe de enfermagem atuantes na UTI (30\%) e no Bloco Cirúrgico 27\% ${ }^{(13)}$.

Atribui-se a maior ocorrência de faltas às peculiaridades de certos setores, onde os trabalhadores estão mais expostos a transtornos de ordem física, Química e psicológica, como no caso de unidades de tratamento intensivo, Que são setores especializados, Que atendem pacientes graves e de alta complexidade. Nesse sentido, pode haver aumento do risco de agravos à saúde e, conseqüentemente, do absenteísmo relacionado a doenças ${ }^{(13)}$.

Quanto ao absenteísmo relacionado a doenças, na Clínica Médica B, atingiu (12,6\%), permitindo inferir considerações Quanto ao tipo de atendimento prestado nesse setor e a relação com os afastamentos. Nessa Clínica, há leitos para portadores de sofrimento mental e em crise, Que requerem cuidados específicos, tornando o trabalho ainda mais desgastante.

Quanto ao acúmulo de vínculos a Tabela 1 demonstra Que aproximadamente $43,4 \%$ (62) dos funcionários em estudo trabalham exclusivamente naQuele hospital. Outra parcela 55,2\% (79) exerce atividades também em outra instituição e 1,4\% (2), trabalham em mais duas instituições.

O fato de Que os funcionários Que mantêm duplo vínculo empregatício podem faltar mais ao trabalho, foi mostrado nos estudos de Nascimento et $\mathrm{a}^{(9)}$, Silva e Marziale ${ }^{(1)}$ e Reis e La Rocca $^{(13)}$ Que procuraram relacionar fatores implicados na ocorrência de absenteísmo entre trabalhadores. E entre tais estudos, destaca-se uma pesquisa realizada em uma Unidade Básica e Distrital de Saúde do Município de Riberão Preto - São Paulo. Nesse estudo, verificou-se um maior número de atestados médicos e licenças entre os funcionários Que trabalhavam também em mais de uma instituição, ou seja, 56\% dos trabalhadores Que mantinham duplo vínculo empregatício faltavam mais ao trabalho por motivo de doença, o que pode ser justificado pelo cansaço físico, estresse mental e pelo comprometimento do repouso necessário ${ }^{(9)}$. Nessa pesquisa, verificou-se que 43,4\% dos trabalhadores que faltaram no período estudado também mantinham vínculo com outras instituições. Assim, é possível relacionar a manutenção de duplo vínculo empregatício como causa do absenteísmo por doença.

A Tabela 4 apresenta a identificação das doenças, segundo agrupamento do CID, Que se mostraram mais freqüentes nos atestados e licenças médicas Que justificavam o absenteísmo dos 143 funcionários participantes da pesquisa. 
De acordo com os dados da Tabela 4, observa-se Que a maior causa do absenteísmo relacionado a doenças dos trabalhadores do sexo masculino e feminino, foi constituída por doenças do sistema ósteo-muscular e conjuntivo, sendo 23,5\% (1 19) entre as mulheres e 28,6\% (16) entre os homens. As doenças do aparelho respiratório, $14 \%$ (75) representam a segunda causa de afastamento entre as mulheres, seguidas das doenças do aparelho circulatório 8,8\% (45). Entre os homens, também as doenças do aparelho respiratório 10,8\% (6), aparecem como a segunda causa do absenteísmo relacionado a doenças, juntamente com os fatores Que induziram a procura de serviço de saúde não especializado 10,8\% (6).

Em estudo realizado sobre a saúde física e mental dos profissionais de enfermagem da Unidade Neonatal de um hospital geral, verificou-se Que o estresse no trabalho ocorre devido ao excesso de responsabilidades, da ausência de reconhecimento da enfermagem como atividade essencial e da falta de autonomia para a tomada de decisões. Além disso, a monotonia gerada pelo trabalho repetitivo provoca sinais de irritabilidade, fadiga, raiva e angústia, Que podem culminar em sintomas, como dor epigástrica e desconforto do aparelho ósteo-muscular ${ }^{(14)}$.

\section{CONCLUSÕES}

A análise dos dados permitiu elaborar considerações referentes ao problema e às hipóteses formuladas neste estudo. Ao analisar a distribuição de eventos sobre falta devido a afastamento, segundo as doenças mais freqüentes nos atestados e licenças médicas, comprovou-se a primeira hipótese, pois os problemas relacionados ao sistema ósteo-muscular apareceram como a primeira causa do absenteísmo. A segunda hipótese - de Que os funcionários com duplo ou tríplice vínculo empregatício se afastam mais do trabalho - também se confirmou.

A terceira hipótese - de Que os funcionários Que trabalham no período noturno, faltariam mais ao trabalho - foi refutada. Evidenciou-se maior incidência de afastamentos entre os funcionários que trabalhavam no período diurno. A quarta hipótese foi confirmada. A maioria dos afastamentos ocorreu entre as funcionárias do sexo feminino. Ao relacionar o afastamento do trabalho e a variável categoria profissional, foi possível confirmar a Quinta hipótese: os trabalhadores de nível fundamental e médio afastam-se mais do trabalho do Que os de nível superior.

Os resultados desta investigação permitiram descrever a situação dos afastamentos do trabalho na equipe de enfermagem do hospital cenário da pesQuisa e poderão subsidiar a direção na definição de políticas direcionadas ao trabalhador, para garantir a Qualidade do cuidado e da vida dos profissionais.

Sugere-se Que outros estudos sejam realizados sobre o tempo de trabalho na instituição, o modelo organizacional e a motivação para o trabalho, como causas do absenteísmo relacionado ao adoecimento, a fim de se atenuar seus efeitos negativos na Qualidade da assistência de enfermagem. Sugere-se, também, a criação de um banco de dados, a ser instalado no hospital cenário, para otimizar o registro das faltas, para acompanhamento e avaliação e permitir outras pesquisas.

\section{REFERÊNCIAS}

1. Alves M.. Causa de Absenteísmo entre auxiliares de enfermagem: uma dimensão do sofrimento no trabalho [tese]. São Paulo (SP): Escola de Enfermagem da USP, Universidade de São Paulo; 1996.

2. Rezende MP. Agravos à saúde de auxiliares de enfermagem resultantes da exposição ocupacional aos riscos físicos [dissertação]. Ribeirão Preto: Escola de Enfermagem da USP, Universidade de São Paulo; 2003.

3. Alves $\mathrm{M}$, Godoy SCB. Procura pelo serviço de atenção à saúde do trabalhador e absenteísmo - doença em um hospital universitário. Rev Min Enferm 2001; 5(1): 73-81.

4. Lunardi Filho WD. Prazer e sofrimento no trabalho: contribuições à organização do processo de trabalho de enfermagem. Rev Bras Enferm 1997; 50(1): 77-92.

5. Dejours C. A loucura do trabalho: estudo de psicopatologia do trabalho. $5^{\text {a }}$ ed. São Paulo: Cortez-Oboré; 2000.

6. Chiavenato I. Recursos humanos edição compacta. $7^{\mathrm{a}}$ ed. São Paulo: Atlas; 2002.

7. Maia MFM. Eficácia das medidas preventivas em saúde ocupacional praticadas pela Universidade Estadual de Montes Claros na biblioteca Prof. Antônio Jorge [dissertação]. Brasília: Universidade Católica de Brasília; 2002.
8. Ministério do Trabalho (BR). Legislação normas da Consolidação das Leis Tabalhistas-CLT. [citado em 20 ago 2004]. Disponível em: www.ministeriodotrabalhoeem prego.org.

9. Nascimento GM. Estudo do absenteísmo dos trabalhadores de enfermagem em uma Unidade Básica e Distrital de saúde do município de Ribeirão Preto [dissertação]. São Paulo (SP): Escola de Enfermagem da USP, Universidade de São Paulo; 2003.

10. Belém JHR, Gaidzinski RR. Estudo das ausências da equipe de enfermagem. Rev Bras Enferm 1998; 5 I (4): 697-708.

11. Mendes R. Patologia do trabalho atualizada e ampliada. $2^{\text {a }}$ ed. Atheneu. São Paulo (SP): Atheneu; 2003.

12. Silva DMPP, Marziale MHP. Absenteísmo de trabalhadores de enfermagem em um hospital universitário. Rev Latino-am Enferm 2000; 8(5): 44-51.

13. Reis RI, La Rocca PF. Fatores relacionados ao absenteísmo por doença em profissionais de enfermagem. Rev Saúde Pública 2003; 37(5): 616-723.

14. Hoga LAK. Causas de estresse e mecanismos de promoção do bem-estar dos profissionais de enfermagem de unidade neonatal. Acta Paul Enferm 2002; 15(2): 18-25. 